\title{
Prognostic nutritional index predicts prognosis in patients with metastatic nasopharyngeal carcinoma
}

This article was published in the following Dove Press journal:

OncoTargets and Therapy

28 September 2016

Number of times this article has been viewed

\author{
Gan-Bao Wei \\ Yao-Yong Lu \\ Rong-Wei Liao \\ Qing-Sheng Chen \\ Kun-Qiang Zhang
}

Department of Radiation Oncology,

People's Hospital of Gaozhou,

Guangdong, People's Republic of

China
Correspondence: Gan-Bao Wei Department of Radiation Oncology, People's Hospital of Gaozhou, No 89 Xi-guan Road, Guangdong 525200 , People's Republic of China

Tel +86 6686664924

Fax +866686697752

Email weiganbao@।63.com
Objective: This study aimed to investigate the prognostic value of Onodera's prognostic nutritional index (PNI) in patients with metastatic nasopharyngeal carcinoma (NPC).

Methods: A total of 187 patients with metastatic NPC treated with cisplatin-based chemotherapy were retrospectively reviewed. The PNI was calculated using the following formula: serum albumin level (gram per liter) $+0.005 \times$ peripheral lymphocyte count (per cubic millimeter). A receiver operating characteristics curve for overall survival (OS) with the highest Youden index was determined to calculate the best cutoff value of PNI. The relationship between PNI and clinicopathological parameters was compared with the $\chi^{2}$ test. Survival analysis was applied to evaluate the predictive value of PNI.

Results: The median PNI in this study was 49.0 (ranging from 32.2 to 78.4). The best cutoff value of PNI for OS was 51.0 according to the receiver operating characteristics analysis. The median OS time was 13.0 months. The multivariate analysis indicated that the complete response (hazard ratio $0.681,95 \%$ confidence interval $0.574-0.902 ; P=0.013$ ) and PNI (hazard ratio 1.732, $95 \%$ confidence interval $1.216-2.892 ; P=0.005)$ were independent prognostic factors for OS in patients with metastatic NPC.

Conclusion: This study revealed that PNI is a simple and effective predictor for overall survival in patients with metastatic NPC.

Keywords: nasopharyngeal carcinoma, inflammation, prognostic nutritional index, prognosis

\section{Introduction}

Nasopharyngeal carcinoma (NPC) is different from other head and neck cancers owing to its epidemiology, pathology, and clinical attributes, with high incidence rates of 20-30 per 100,000 population being reported in some areas of Southeast Asia and southern China. ${ }^{1,2}$ Most of the NPC patients had advanced disease at diagnosis owing to its unusual anatomic location and the aggressive behavior of poorly differentiated pathologic type. ${ }^{3}$ Although advances in radiotherapeutic techniques, diagnostic imaging, and chemotherapy regimens improved the cure rate recently, distant metastases were still the main reason for the failure of treatment. ${ }^{4}$ However, even within the same TNM stage, there is still wide variability in clinical response and patient prognosis. ${ }^{2}$ It is therefore paramount to develop a reliable and easily available model to identify high-risk patients that complements conventional TNM staging system.

Recently, there extensive evidence has revealed that systemic inflammation plays an important role in cancer development and progression and is strongly associated with prognosis in patients with various types of neoplasms. ${ }^{5,6}$ The host's systemic 
inflammatory response could be reflected by fluctuations of the circulating concentrations of acute-phase proteins and other cellular components. ${ }^{7}$ The Onodera's prognostic nutritional index (PNI), first proposed by Onodera et al, is a novel systemic inflammation-based prognostic score that combines lymphocyte count and serum albumin level. ${ }^{7,8}$ Previous studies have demonstrated that the PNI was associated with survival in several types of malignancies. ${ }^{9-15}$ However, to the best of our knowledge, there is no study regarding the prognostic role of PNI in metastatic NPC. In this study, we therefore aimed to examine the clinical value of PNI for metastatic NPC patients and to evaluate the correlation between the PNI and clinicopathological factors.

\section{Materials and methods}

\section{Ethics statement}

Written informed consent was obtained from all participants and this study was approved by the Ethics Committee of People's Hospital of Gaozhou, Guangdong, People's Republic of China. This study was conducted in accordance with the principles of the Declaration of Helsinki.

\section{Patient characteristics}

From January 2003 to June 2013, the inpatient medical records of 187 patients in People's Hospital of Gaozhou who were histologically diagnosed as having metastatic NPC treated with first-line cisplatin-based chemotherapy were respectively reviewed. According to the seventh edition of the American Joint Commission on Cancer staging system, these patients with distant metastasis were staged as Stage IVC. All included patients met the following criteria: 1) histologically confirmed NPC, 2) at least one radiologically measurable disease, 3) Karnofsky performance score (KPS) $\geq 60$, and 4) normal renal and liver function. The exclusion criteria were the following: 1) history of other malignancies and 2) patients with clinical evidence of infection or other inflammatory conditions.

The demographics and clinicopathlogical characteristics such as sex, age, KPS, and chemotherapy regimen were retrospectively gathered from medical records. Pretreatment whole blood parameters including white blood cells, lymphocytes, neutrophils, hemoglobin, and platelets; albumin concentration; total bilirubin; and alkaline phosphatase were also obtained. PNI was defined per the following formula: serum albumin level (gram per liter) $+0.005 \times$ peripheral lymphocyte count (per cubic millimeter). ${ }^{7}$ Serum Epstein-Barr virus (EBV) VCA/IgA and EA/IgA antibody levels were determined by titration using an immunoenzymatic method.

\section{Chemotherapy}

All eligible patients were treated with cisplatin-based chemotherapy regimens as the first-line treatment. Of the 187 eligible patients, 97 patients (51.9\%) received PF regimen (cisplatin, $25 \mathrm{mg} / \mathrm{m}^{2}$ intravenously [IV] on Days 1-3 every 3 weeks and 5-fluorouracil, $500 \mathrm{mg} / \mathrm{m}^{2} \mathrm{IV}$ on Days $1-5$ every 3 weeks) and 56 patients (29.9\%) received TP regimen (cisplatin, $25 \mathrm{mg} / \mathrm{m}^{2} \mathrm{IV}$ on Days $1-3$ every 3 weeks and paclitaxel, $175 \mathrm{mg} / \mathrm{m}^{2}$ IV over 3 hours with standard premedication on Day 1 every 3 weeks). The remaining 34 (18.2\%) patients received other regimens. The median number of cycles of chemotherapy was 4 (ranging from 2 to 12 ).

\section{Patient follow-up}

After completion of treatment, patients were regularly followed up every 3 months during the first 2 years and every 6 months thereafter for up to 5 years or until death.

Physical examination, blood test, and imaging studies of the relevant region(s) were performed. The response of treatment was evaluated according to Response Evaluation Criteria in Solid Tumors (RECIST 1.0). The end of follow-up was November 2015.

\section{Statistical analysis}

Continuous variables were presented as mean \pm standard deviation, and the categorical variables were presented as numbers and percentages. A receiver operating characteristics curve for overall survival (OS) was generated to determine the sensitivity and specificity. The highest Youden index was determined to calculate the best cutoff value of PNI. The area under the curve (AUC) was calculated to evaluate the discriminatory ability of PNI, VCA/IgA, and EA/IgA for OS. The chi-square or Fisher's exact test was used to compare the categorical variables. OS was the study end point. Survival analysis was drawn using the Kaplan-Meier method, and the differences were compared using the log-rank test. Multivariate analysis using a Cox proportional hazards model was used to test independent significance. A two-tailed $P$-value $<0.05$ was considered to be statistically significant. Statistical analyses were performed using the SPSS 21.0 software (IBM Corporation, Armonk, NY, USA).

\section{Results \\ Patient characteristics}

The clinicopathological characteristics of the 187 patients are shown in Table 1. The median age at diagnosis of metastatic NPC was 47 years (range 21-75 years), and there was a male predominance $(83.4 \%)$. Ninety-nine patients $(52.9 \%)$ 
Table I Demographic and baseline characteristics of the study population

\begin{tabular}{|c|c|}
\hline Characteristics & No of patients (\%) \\
\hline Age, median (range), years & $47(21-75)$ \\
\hline \multicolumn{2}{|l|}{ Sex } \\
\hline Male & $156(83.4)$ \\
\hline Female & $31(16.6)$ \\
\hline \multicolumn{2}{|l|}{ KPS } \\
\hline$\geq 80$ & |7| (9|.4) \\
\hline$<80$ & $16(8.6)$ \\
\hline \multicolumn{2}{|l|}{ Synchronous metastasis } \\
\hline Present & $32(17.1)$ \\
\hline Absent & $155(82.9)$ \\
\hline \multicolumn{2}{|l|}{ Lung metastasis } \\
\hline Present & $96(51.3)$ \\
\hline Absent & 91 (48.7) \\
\hline \multicolumn{2}{|l|}{ Liver metastasis } \\
\hline Present & $70(37.4)$ \\
\hline Absent & $117(62.6)$ \\
\hline \multicolumn{2}{|l|}{ Bone metastasis } \\
\hline Present & $67(35.8)$ \\
\hline Absent & $120(64.2)$ \\
\hline \multicolumn{2}{|l|}{ Distant nodal metastasis } \\
\hline Present & $2 I(I I .2)$ \\
\hline Absent & $166(88.8)$ \\
\hline BMI, median (range), $\mathrm{kg} / \mathrm{m}^{2}$ & $19.7(\mid 15.7-27.1)$ \\
\hline WBC, $\times 10^{9} / \mathrm{L}$, median (range) & $7.0(3.1-10.9)$ \\
\hline Lymphocyte, $\times 10^{9} / \mathrm{L}$, median (range) & $1.30(0.35-4.7 I)$ \\
\hline Hemoglobin, g/L, median (range) & $130.1(91.0-163.0)$ \\
\hline Platelet, $\times 10^{9} / \mathrm{L}$, median (range) & $23 । .3(97.0-5 \mid I .0)$ \\
\hline Serum albumin, g/L, median (range) & $37.1(30.2-44.7)$ \\
\hline PNI, median (range) & $49.0(32.2-78.4)$ \\
\hline Serum LDH, U/L, median (range) & $24 \mid(73-459)$ \\
\hline
\end{tabular}

Abbreviations: KPS, Karnofsky performance score; BMI, body mass index; WBC, white blood cell; PNI, prognostic nutritional index; LDH, lactate dehydrogenase.

had more than one metastatic site, and lung metastasis was the most common site (51.3\%). The median serum albumin level was $37.1 \mathrm{~g} / \mathrm{L}$ (ranging from 30.2 to $44.7 \mathrm{~g} / \mathrm{L}$ ), and the median lymphocyte count was $1.30 \times 10^{9} / \mathrm{L}$ (ranging from 0.35 to 4.71 ). The median $\mathrm{EA} / \operatorname{IgA}$ and $\mathrm{VCA} / \operatorname{IgA}$ antibody titers were $1: 160$ (ranging from 0 to $1: 1,280$ ) and $1: 20$ (ranging from 0 to 1:80), respectively. The median PNI in this study was 49.0 (ranging from 32.2 to 78.4 ). The best cutoff value of PNI for OS was 51.0 (sensitivity: 0.656 and specificity: 0.781 ) according to the receiver operating characteristics analysis.

\section{Correlation between PNI with clinicopathological characteristics and response of chemotherapy}

Baseline characteristics for each PNI group and comparisons between PNI groups are shown in Table 2. Patients with high PNI (PNI $\geq 51$ ) had a significantly higher body mass
Table 2 Relationship between PNI and relevant characteristics of patients

\begin{tabular}{|c|c|c|c|}
\hline Characteristics & PNI $<5$ I & $P N I \geq 5 I$ & $P$-value \\
\hline Age $(<47 / \geq 47$ years $)$ & $5 \mathrm{I} / 52$ & $44 / 40$ & 0.769 \\
\hline Sex (male/female) & $87 / 16$ & $69 / 15$ & 0.697 \\
\hline KPS $(\geq 90 /<90)$ & $93 / 10$ & $78 / 6$ & 0.606 \\
\hline $\operatorname{BMI}\left(<19.7 / \geq 19.7 \mathrm{~kg} / \mathrm{m}^{2}\right)$ & $44 / 59$ & $51 / 33$ & 0.019 \\
\hline Number of involved sites $(I />I)$ & $52 / 51$ & $36 / 48$ & 0.307 \\
\hline $\begin{array}{l}\text { Synchronous metastasis } \\
\text { (present/absent) }\end{array}$ & $17 / 86$ & $14 / 69$ & 0.947 \\
\hline Lung metastasis (present/absent) & $47 / 56$ & $49 / 35$ & 0.106 \\
\hline Bone metastasis (present/absent) & $33 / 70$ & $34 / 50$ & 0.283 \\
\hline Liver metastasis (present/absent) & $34 / 69$ & $36 / 48$ & 0.175 \\
\hline $\begin{array}{l}\text { Distant nodal metastasis } \\
\text { (present/absent) }\end{array}$ & $7 / 96$ & $14 / 70$ & 0.038 \\
\hline WBC, $\times 10^{9}$ counts $/ L(<5.3 / \geq 5.3)$ & $59 / 44$ & $36 / 48$ & 0.057 \\
\hline Hemoglobin, $g / L(<|27| \geq \mid 27)$ & $47 / 56$ & $47 / 37$ & 0.187 \\
\hline Platelet, $\times 10^{9}$ counts $/ L(<215 / \geq 215)$ & $48 / 55$ & $43 / 41$ & 0.559 \\
\hline AST, U/L $(<24 / \geq 24)$ & $52 / 51$ & $42 / 42$ & 0.947 \\
\hline $\mathrm{ALT}, \mathrm{U} / \mathrm{L}(<2 \mathrm{I} / \geq 2 \mathrm{I})$ & $54 / 49$ & $43 / 41$ & 0.884 \\
\hline Serum LDH, U/L $(<24 I / \geq 24 I)$ & $49 / 54$ & $45 / 39$ & 0.463 \\
\hline
\end{tabular}

Abbreviations: PNI, prognostic nutritional index; KPS, Karnofsky performance score; BMI, body mass index; WBC, white blood cell; AST, aspartate aminotransferase; ALT, alanine aminotransferase; LDH, lactate dehydrogenase.

index $(P=0.019)$ and a higher percentage of distant nodal metastasis $(P=0.038)$ in comparison with patients with low PNI (PNI $<51)$. There was a trend toward an association of PNI with white blood cell $(P=0.057)$.

To evaluate the impact of PNI on the response of palliative chemotherapy, we also analyzed the relationship between neutrophil-to-lymphocyte ratio and response rate. In patients with PNI $\geq 51$, two patients achieved complete response (CR), 60 patients achieved partial response (PR), 12 patients were found to have with stable disease (SD), and 10 patients were evaluated as having progressive disease (PD). In patients whose PNI was $<51$, three patients achieved CR, 68 patients achieved PR, 16 patients were found to have with SD, and 16 patients were evaluated as having $\mathrm{PD}$ (Figure 1). The $\mathrm{CR}$ rate $(P=0.831)$, response $(\mathrm{CR}+\mathrm{PR})$ rate $(P=0.518)$, and the disease control $(\mathrm{CR}+\mathrm{PR}+\mathrm{SD})$ rate $(P=0.529)$ were similar between the two groups.

\section{Survival analyses}

During the follow-up, a total of 131 (70.1\%) patients had died, and the median OS time was 13.0 months. The potential prognostic variables including age, sex, KPS, number of involved sites, synchronous metastasis, metastasis sites (lung, bone, liver, and distant lymph node), chemotherapy regimen, CR, PNI, and related serum markers were analyzed. Results of univariate and multivariate analyses for OS are depicted in Table 3. Univariate analysis revealed that number 


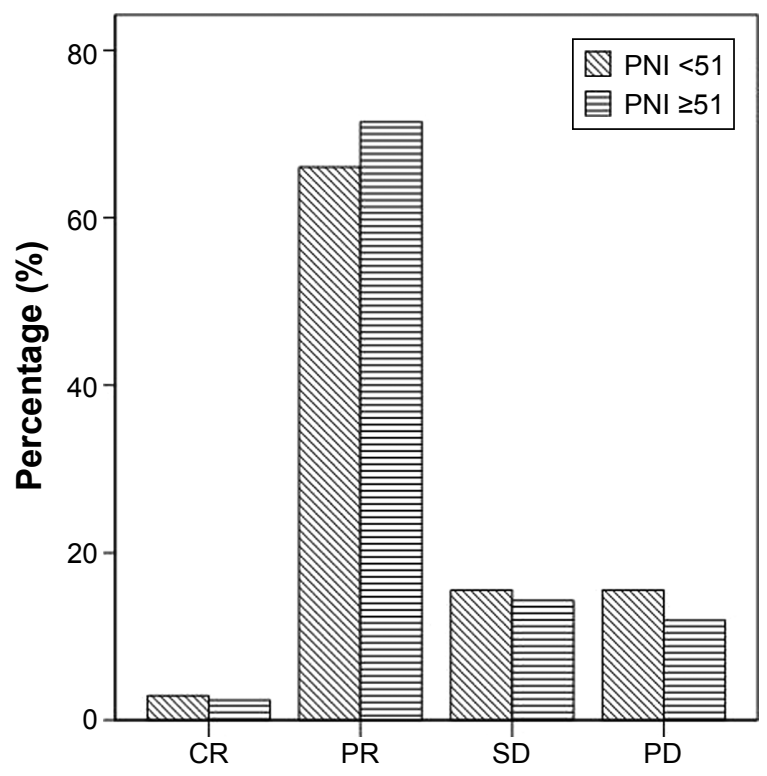

Figure I Correlations of $\mathrm{PNI}$ and tumor response of chemotherapy. Abbreviations: PNI, prognostic nutritional index; $C R$, complete response; PR, partial response; SD, stable disease; $P D$, progressive disease.

of involved sites $(P=0.039), \mathrm{CR}(P=0.011), \mathrm{VCA} / \mathrm{IgA}$ titers $(P=0.036)$, and PNI $(P=0.002)$ were significantly prognostic factors for OS. To identify independent prognostic factors for OS, the factors that were statistically significant on univariate analysis $(P<0.10)$ were then subjected to multivariate analysis. The multivariate analysis indicated that the $\mathrm{CR}$ (hazard ratio $0.681,95 \%$ confidence interval $0.574-0.902$; $P=0.013$ ) and PNI (hazard ratio 1.732, 95\% confidence interval 1.216-2.892; $P=0.005)$ were independent prognostic factors for OS (Table 3; Figure 2).

The AUC was calculated to evaluate the discriminatory ability of PNI, VCA/IgA, and EA/IgA for OS. The PNI had a higher AUC value (0.728) in comparison with $\mathrm{VCA} / \mathrm{IgA}$ titers $(0.652 ; P=0.048)$ and $\mathrm{EA} / \operatorname{IgA}$ titers $(0.563 ; P=0.027)$, as is shown in Figure 3.

\section{Discussion}

To the best of our knowledge, this was the first study to explore the prognostic value of PNI in metastatic NPC patients. This study, consisting of 187 metastatic NPC patients, revealed that PNI was significantly associated with poor prognosis in patients with metastatic NPC. Besides determining a prognostic value of PNI for OS, our data also demonstrated a significant independent association between PNI and clinicopathologic parameters.

Variations among the outcomes of patients with various malignancies are not solely determined by the characteristics of malignancies but also by host response factors. ${ }^{5,16}$ It has been widely accepted that the systemic inflammatory response is an important regulator in the oncogenesis, tumor development, and metastasis by recruiting regulatory $\mathrm{T}$ lymphocytes and circulating cytokines, including circulating interleukin-1,

Table 3 Univariate and multivariate analyses of prognostic factors for OS

\begin{tabular}{|c|c|c|c|c|}
\hline \multirow[t]{2}{*}{ Variables } & \multicolumn{2}{|c|}{ Univariate analysis } & \multicolumn{2}{|c|}{ Multivariate analysis } \\
\hline & $P$-value & HR (95\% Cl) & $P$-value & HR (95\% CI) \\
\hline Age $(<47 / \geq 47$ years $)$ & 0.211 & $1.119(0.881-1.652)$ & & \\
\hline Sex (male/female) & 0.089 & $1.496(0.916-1.658)$ & 0.136 & $\mathrm{I} .463(0.885-1.532)$ \\
\hline KPS $(\geq 90 /<90)$ & 0.385 & $0.723(0.348-1.503)$ & & \\
\hline BMI $(<19.7 / \geq 19.7$ kg/m²) & 0.803 & $1.034(0.796-1.342)$ & & \\
\hline Number of involved sites $(>I / I)$ & 0.039 & $1.685(1.049-2.766)$ & 0.335 & I.379 (0.758-I.763) \\
\hline Synchronous metastasis (present/absent) & 0.083 & $1.377(0.796-1.942)$ & 0.149 & $1.129(0.843-1.362)$ \\
\hline Lung metastasis (present/absent) & 0.254 & $1.225(0.842-1.698)$ & & \\
\hline Bone metastasis (present/absent) & 0.342 & $0.723(0.348-1.503)$ & & \\
\hline Liver metastasis (present/absent) & 0.195 & $1.373(0.863-1.782)$ & & \\
\hline Distant nodal metastasis (present/absent) & 0.259 & $0.785(0.652-1.623)$ & & \\
\hline Chemotherapy regimen (TP/PF/others) & 0.136 & $1.273(0.865-1.847)$ & & \\
\hline Complete response (yes/no) & 0.011 & $0.623(0.433-0.897)$ & 0.013 & $0.68 \mathrm{I}(0.574-0.902)$ \\
\hline WBC, $\times 10^{9}$ counts $/ L(<5.3 / \geq 5.3)$ & 0.142 & $1.373(0.824-1.685)$ & & \\
\hline Hemoglobin, g/L $(<|27| \geq \mid 27)$ & 0.231 & $1.182(0.736-1.596)$ & & \\
\hline Platelet, $\times 10^{9}$ counts $/ L(<2|5 / \geq 2| 5)$ & 0.756 & $1.068(0.876-1.258)$ & & \\
\hline Serum LDH, U/L $(<24 I / \geq 24 I)$ & 0.876 & $0.978(0.77 \mathrm{I}-\mathrm{I} .196)$ & & \\
\hline $\mathrm{PNI}(<5 \mathrm{I} / \geq 5 \mathrm{I})$ & 0.002 & $1.845(1.246-2.73 \mid)$ & 0.005 & $1.732(1.216-2.892)$ \\
\hline$E A / \lg A$ titers $(\geq 1: 20 /<1: 20)$ & 0.268 & $\mathrm{I} .045(0.78 \mathrm{I}-\mathrm{I} .358)$ & & \\
\hline VCA/lgA titers $(\geq 1: 160 /<1: 160)$ & 0.036 & $1.358(1.069-1.964)$ & 0.187 & $1.119(0.864-1.485)$ \\
\hline
\end{tabular}

Abbreviations: OS, overall survival; HR, hazard ratio; $95 \% \mathrm{Cl}, 95 \%$ confidence interval; KPS, Karnofsky performance score; BMI, body mass index; WBC, white blood cell; LDH, lactate dehydrogenase; TP, paclitaxel + cisplatin; PF, cisplatin + 5-fluorouracil; PNI, prognostic nutritional index; EA, early antigen; VCA, viral capsid antigen; IgA, immunoglobulin A. 


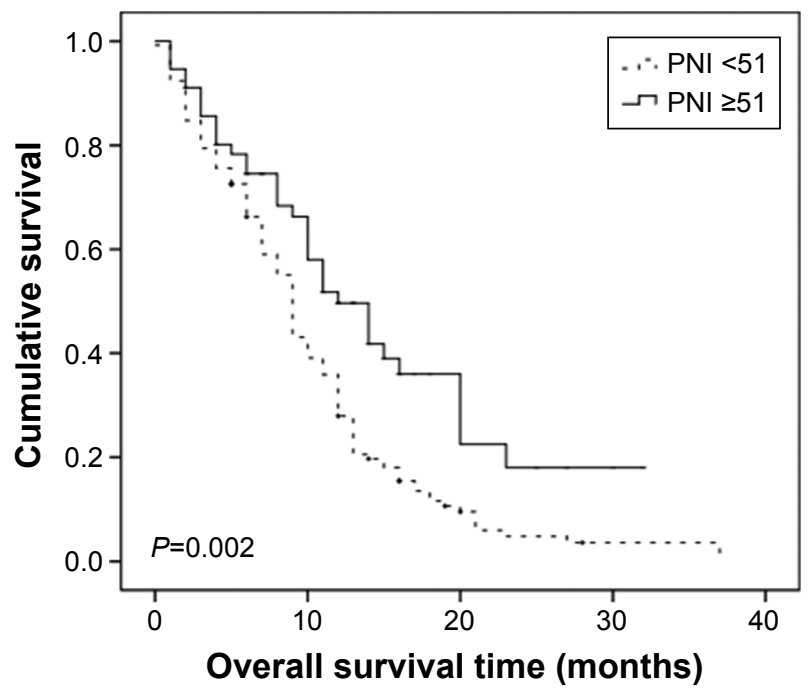

Figure 2 Kaplan-Meier curves estimates for OS according to PNI. Abbreviations: OS, overall survival; $\mathrm{PNI}$, prognostic nutritional index.

interleukin-6, and tumor necrosis factor alpha. ${ }^{17}$ Additionally, EBV infection is associated with NPC in endemic areas and could induce the consistent release of viral immunogenic proteins that lead to a potent immune response. ${ }^{18}$ Plasma EBV DNA load has been identified to have a prognostic role in metastatic NPC patients. ${ }^{19}$ In patients with malignancies, the presence of systemic inflammation could be reflected by inflammation-based prognostic scores, including platelet-tolymphocyte ratio, neutrophil-to-lymphocyte ratio, modified Glasgow prognostic score, and PNI. ${ }^{20}$ The original purpose of PNI was to evaluate the nutritional and immunity status

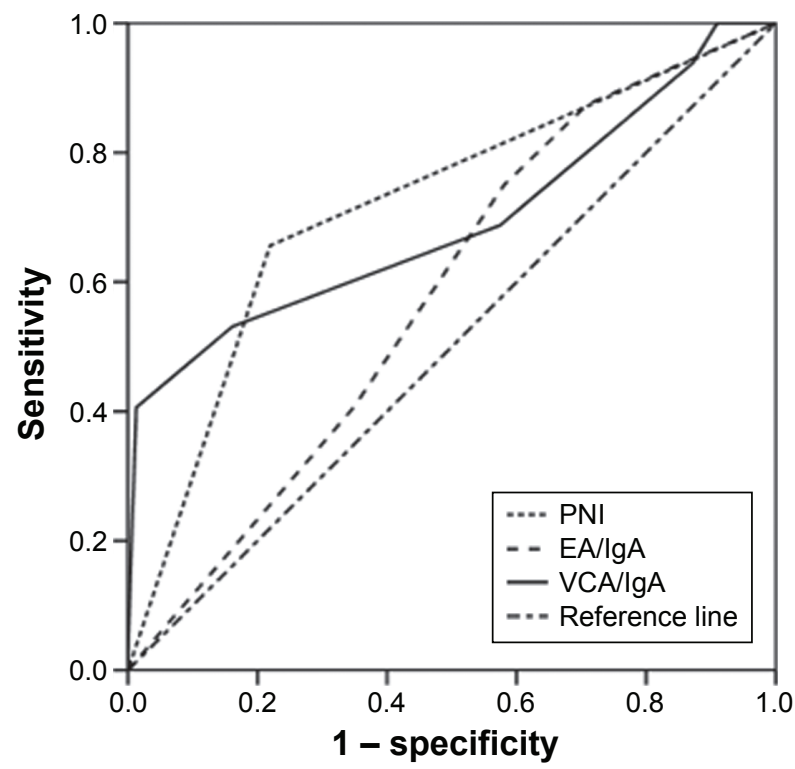

Figure 3 The receiver operating curves for OS prediction among PNI, VCA/lgA, and $\mathrm{EA} / \lg A$.

Abbreviations: $\mathrm{PNI}$, prognostic nutritional index; OS, overall survival. to predict short-term postoperative outcome of patients with gastrointestinal cancers. ${ }^{17}$ PNI based on lymphocyte count and serum albumin level reflects systemic immunity and nutritional status. Recently, accumulating evidence indicated that PNI had a prognostic role in the long-term survival outcome of patients with several malignancies, including pancreatic cancer, gastric carcinoma, esophageal cancer, colorectal cancer, malignant pleural mesothelioma, non-small-cell lung cancer, hepatocellular carcinoma, and renal cell cancer. ${ }^{10-15,21}$ Hong et $\mathrm{al}^{22}$ revealed that patients with low PNI had a worse median OS time and that PNI was an independent significant prognostic factor for OS. In this study, the multivariate Cox analysis demonstrated that PNI was an independent prognostic factor for patients with metastatic NPC.

The mechanisms that lower PNI and decrease survival in metastatic NPC patients would be influenced by many confounding factors. PNI is calculated from lymphocyte count and serum albumin level. Serum albumin was confirmed as being associated with systemic inflammation through high levels of proinflammatory cytokines and growth factors. Albumin may help to stabilize cell growth and DNA replication, buffer various biochemical changes, and maintain sex hormone homeostasis and calcium level, thus demonstrating anticancer effects. ${ }^{23}$ In vitro, the proliferation or growth of cancer cell lines could be modulated by high albumin concentrations. ${ }^{24}$ In addition, malnutrition and cachexia could be partially reflected by hypoalbuminemia, and a low level of albumin could weaken a number of human defense mechanisms, including anatomic barriers, cellular and humoral immunity, and phagocyte function, thus diminishing compliance with treatment and increasing susceptibility to infection. ${ }^{25,26}$ Furthermore, malnutrition and inflammation could suppress albumin synthesis in advanced stages in cancer patients. ${ }^{27}$ Gupta et $\mathrm{al}^{28}$ summarized 29 studies and revealed that lower pretreatment serum albumin levels were associated with poor outcome in cancer patients. On the other hand, lymphocytes are crucial components of the adaptive immune system and could be suppressed in malignancies by several pathways, including inhibition of regulatory T-cells and tumor-associated macrophages infiltration, dendritic cell differentiation, and activation. ${ }^{29}$ Infiltrating lymphocytes could represent an effective anticancer cellular immune response, and high peripheral lymphocyte percentage was also significantly associated with a better prognosis in NPC patients. ${ }^{29}$ In addition, reduced peripheral lymphocyte count indicated the impairment of innate cellular immunity and was associated with disease severity and poor prognosis. ${ }^{29,30}$ 
Notably, there is an amount of ongoing research into the effect of nonsteroidal anti-inflammatory drugs on antitumor treatment, including lung cancer, colon cancer, esophagus cancer, etc. ${ }^{31-33}$ Also, the PNI, as an inexpensive, reliable, and easily available marker, may be of importance to select patients who might be candidates for modulation of systemic inflammation and could provide a well-defined therapeutic target for future clinical studies. Accordingly, it is also of interest to investigate the modification of the systemic inflammatory response in metastatic NPC patients.

Notably, CR was an independent prognostic factor for OS in this study, which is consistent with previous studies. Jin et $\mathrm{al}^{34}$ reported that $\mathrm{CR}$ was an independent prognostic factor in metastatic NPC patients receiving chemotherapy. In addition, those patients who were evaluated as having $\mathrm{SD} / \mathrm{PD}$ might develop clinical drug resistance that leads to treatment failure and poorer OS compared with patients who achieved CR. ${ }^{35}$ Several molecular targets that were associated with chemoresistance have identified as negative prognostic factors of OS in patients with metastatic NPC receiving chemotherapy, including multidrug resistance 1 , multidrug resistance-associated protein-1, and drug-resistance gene annexin $\mathrm{I}^{35,36}$ Besides, Liu et $\mathrm{al}^{37}$ reported that the unsatisfactory tumor response (SD/PD) after neoadjuvant chemotherapy predicted poor prognosis in advanced-stage NPC patients.

This study was unavoidably limited by its retrospective nature and relatively small number of metastatic NPC patients in a single institution. In addition, PNI was only assessed at a single time point before the treatment. The relationship between the kinetics of PNI and its prognostic effect in NPC patients would be of interest. Further research is needed to confirm these preliminary results.

\section{Conclusion}

This study revealed that PNI is a simple and effective predictor for OS in patients with metastatic NPC.

\section{Disclosure}

The authors report no conflicts of interest in this work.

\section{References}

1. Cao SM, Simons MJ, Qian CN. The prevalence and prevention of nasopharyngeal carcinoma in China. Chin J Cancer. 2011;30(2):114-119.

2. Wei WI, Sham JS. Nasopharyngeal carcinoma. Lancet. 2005;365(9476): 2041-2054.

3. Lee AW, Lau WH, Tung SY, et al. Preliminary results of a randomized study on therapeutic gain by concurrent chemotherapy for regionallyadvanced nasopharyngeal carcinoma: NPC-9901 Trial by the Hong Kong Nasopharyngeal Cancer Study Group. J Clin Oncol. 2005;23(28): 6966-6975.
4. Tao Y, Bidault F, Bosq J, et al. Distant metastasis of undifferentiated carcinoma of nasopharyngeal type. Onkologie. 2008;31(11):574-575.

5. Muliawati Y, Haroen H, Rotty LW. Cancer anorexia - cachexia syndrome. Acta Med Indones. 2012;44(2):154-162.

6. McMillan DC. Systemic inflammation, nutritional status and survival in patients with cancer. Curr Opin Clin Nutr Metab Care. 2009;12(3): 223-226.

7. Proctor MJ, Morrison DS, Talwar D, et al. A comparison of inflammationbased prognostic scores in patients with cancer. A Glasgow Inflammation Outcome Study. Eur J Cancer. 2011;47(17):2633-2641.

8. Onodera T, Goseki N, Kosaki G. Prognostic nutritional index in gastrointestinal surgery of malnourished cancer patients. Nihon Geka Gakkai Zasshi. 1984;85(9):1001-1005.

9. Nozoe T, Ninomiya M, Maeda T, et al. Prognostic nutritional index: a tool to predict the biological aggressiveness of gastric carcinoma. Surg Today. 2010;40(5):440-443.

10. Mohri $Y$, Inoue $Y$, Tanaka K, et al. Prognostic nutritional index predicts postoperative outcome in colorectal cancer. World J Surg. 2013;37(11): 2688-2692.

11. Hofbauer SL, Pantuck AJ, de Martino M, et al. The preoperative prognostic nutritional index is an independent predictor of survival in patients with renal cell carcinoma. Urol Oncol. 2015;33(2):68.e61-67.

12. Pinato DJ, North BV, Sharma R. A novel, externally validated inflammation-based prognostic algorithm in hepatocellular carcinoma: the prognostic nutritional index (PNI). Br J Cancer. 2012;106(8): 1439-1445.

13. Kinoshita A, Onoda H, Imai N, et al. Comparison of the prognostic value of inflammation-based prognostic scores in patients with hepatocellular carcinoma. Br J Cancer. 2012;107(6):988-993.

14. Yao ZH, Tian GY, Wan YY, et al. Prognostic nutritional index predicts outcomes of malignant pleural mesothelioma. J Cancer Res Clin Oncol. 2013;139(12):2117-2123.

15. Kanda M, Fujii T, Kodera Y, et al. Nutritional predictors of postoperative outcome in pancreatic cancer. Br J Surg. 2011;98(2):268-274.

16. Lis CG, Gupta D, Lammersfeld CA, et al. Role of nutritional status in predicting quality of life outcomes in cancer - a systematic review of the epidemiological literature. Nutr J. 2012;11:27.

17. Jian-Hui C, Iskandar EA, Cai S, et al. Significance of Onodera's prognostic nutritional index in patients with colorectal cancer: a large cohort study in a single Chinese institution. Tumour Biol. 2016;37(3):3277-3283.

18. Lin JC, Wang WY, Chen KY, et al. Quantification of plasma EpsteinBarr virus DNA in patients with advanced nasopharyngeal carcinoma. N Engl J Med. 2004;350(24):2461-2470.

19. An X, Wang FH, Ding PR, et al. Plasma Epstein-Barr virus DNA level strongly predicts survival in metastatic/recurrent nasopharyngeal carcinoma treated with palliative chemotherapy. Cancer. 2011;117(16): $3750-3757$.

20. Roxburgh CS, McMillan DC. Role of systemic inflammatory response in predicting survival in patients with primary operable cancer. Future Oncol. 2010;6(1):149-163.

21. Sheng J, Yang YP, Ma YX, et al. Low prognostic nutritional index correlates with worse survival in patients with advanced NSCLC following EGFR-TKIs. PLoS One. 2016;11(1):e0147226.

22. Hong S, Zhou T, Fang W, et al. The prognostic nutritional index (PNI) predicts overall survival of small-cell lung cancer patients. Tumour Biol. 2015;36(5):3389-3397.

23. Seaton K. Albumin concentration controls cancer. J Natl Med Assoc. 2001;93(12):490-493.

24. Laursen I, Briand P, Lykkesfeldt AE. Serum albumin as a modulator on growth of the human breast cancer cell line, MCF-7. Anticancer Res. 1990;10(2A):343-351.

25. Scott HR, McMillan DC, Forrest LM, et al. The systemic inflammatory response, weight loss, performance status and survival in patients with inoperable non-small cell lung cancer. Br J Cancer. 2002;87(3): 264-267.

26. Chandra RK. Nutrition and immunology: from the clinic to cellular biology and back again. Proc Nutr Soc. 1999;58(3):681-683. 
27. Ballmer PE, Ochsenbein AF, Schutz-Hofmann S. Transcapillary escape rate of albumin positively correlates with plasma albumin concentration in acute but not in chronic inflammatory disease. Metabolism. 1994; 43(6):697-705.

28. Gupta D, Lis CG. Pretreatment serum albumin as a predictor of cancer survival: a systematic review of the epidemiological literature. Nutr J. 2010;9:69.

29. He JR, Shen GP, Ren ZF, et al. Pretreatment levels of peripheral neutrophils and lymphocytes as independent prognostic factors in patients with nasopharyngeal carcinoma. Head Neck. 2012;34(12):1769-1776.

30. Ray-Coquard I, Cropet C, Van Glabbeke M, et al. Lymphopenia as a prognostic factor for overall survival in advanced carcinomas, sarcomas, and lymphomas. Cancer Res. 2009;69(13):5383-5391.

31. Gridelli C, Gallo C, Ceribelli A, et al. Factorial phase III randomised trial of rofecoxib and prolonged constant infusion of gemcitabine in advanced non-small-cell lung cancer: the GEmcitabine-COxib in NSCLC (GECO) study. Lancet Oncol. 2007;8(6):500-512.

32. Fuchs CS, Ogino S. Aspirin therapy for colorectal cancer with PIK3CA mutation: simply complex! J Clin Oncol. 2013;31(34):4358-4361.
33. Szumilo J, Burdan F, Szumilo M, et al. Cyclooxygenase inhibitors in chemoprevention and treatment of esophageal squamous cell carcinoma. Pol Merkur Lekarski. 2009;27(161):408-412.

34. Jin Y, Ye X, He C, Zhang B, Zhang Y. Pretreatment neutrophil-tolymphocyte ratio as predictor of survival for patients with metastatic nasopharyngeal carcinoma. Head Neck. 2015;37(1):69-75.

35. Hsu CH, Chen CL, Hong RL, et al. Prognostic value of multidrug resistance 1, glutathione-S-transferase-pi and p53 in advanced nasopharyngeal carcinoma treated with systemic chemotherapy. Oncology. 2002; 62(4):305-312.

36. Chow BH, Chua DT, Sham JS, et al. Increased expression of annexin I is associated with drug-resistance in nasopharyngeal carcinoma and other solid tumors. Proteomics Clin Appl. 2009;3(6):654-662.

37. Liu LT, Tang LQ, Chen QY, et al. The prognostic value of plasma epstein-barr viral DNA and tumor response to neoadjuvant chemotherapy in advanced-stage nasopharyngeal carcinoma. Int $J$ Radiat Oncol Biol Phys. 2015;93(4):862-869.

\section{Publish your work in this journal}

OncoTargets and Therapy is an international, peer-reviewed, open access journal focusing on the pathological basis of all cancers, potential targets for therapy and treatment protocols employed to improve the management of cancer patients. The journal also focuses on the impact of management programs and new therapeutic agents and protocols on

\section{Dovepress}

patient perspectives such as quality of life, adherence and satisfaction The manuscript management system is completely online and includes a very quick and fair peer-review system, which is all easy to use. Visit http://www.dovepress.com/testimonials.php to read real quotes from published authors. 